\title{
Qualidade de vida dos profissionais de saúde dos programas de residências multidisciplinares
}

Quality of life of health professionals of multidisciplinary residency programs

Calidad de vida de los profesionales de salud de los programas de residencias multidisciplinares

\section{Suely Sueko Viski Zaneii, Raquel Araujo Oliveirai Iveth Yamaguchi Whitakeriii}

\begin{abstract}
Resumo: Objetivos: caracterizar os profissionais dos programas de Residência multiprofissional quanto aos dados sociodemográficos, educacionais e rotinas de vida diária como sono e hábitos alimentares e avaliar sua qualidade de vida $(\mathrm{QV})$. Método: estudo descritivo, transversal, quantitativo realizado em uma universidade pública no início de 2014. O instrumento utilizado para avaliação da qualidade de vida foi $o$ World Health Organization Quality Of Life-bref (WHOQOL-bref), aplicado por meio de plataforma eletrônica. Resultados: a confiabilidade do instrumento variou de 0,63-0,79. Participaram $49 \%$ do total de residentes matriculados, a maioria mulheres, com 25 anos, solteiras e procedentes de São Paulo. Os profissionais residentes dormem em média seis horas por noite e referem alimentação mais ou menos saudável. A pontuação da QV no domínio Físico foi de 55,96; Psicológico 56,96; Relações Sociais alcançou 62,76 e Meio Ambiente de 49,65. Conclusão: a avalição do WHOQOL-bref revelou que, na maioria dos domínios, a QV dos residentes é comprometida.
\end{abstract}

Descritores: Qualidade de vida; Estudantes de ciências da saúde; Ocupações em saúde; Capacitação profissional

\begin{abstract}
Aim: to characterize the professionals enrolled in the Multidisciplinary Residency programs according to socio-demographic, educational, and daily living routines data, such as sleep and eating habits, and to assess their quality of life (QoL). Method: descriptive, cross-sectional, quantitative study performed at a public university in the beginning of 2014. The tool used to assess the quality of life was the World Health Organization Quality Of Life-bref (WHOQOL-bref), applied through an electronic platform. Results: the tool's reliability ranged from 0,630,79. Forty-nine percent of residents participated, mostly women, 25 years old, single, from São Paulo. The residents sleep an average of 6 hours per night and refer to eating relatively healthily. The QoL score in the Physical health domain was 55.96; Psychological 56.96; Social Relationships 62.76, and Environment 49.65. Conclusion: The WHOQOL-bref's assessment revealed that, in most domains, the QoL of the residents is compromised.
\end{abstract}

Descriptors: Quality of life; Students, health occupations ; Health occupations; Professional training

\footnotetext{
${ }^{i}$ Enfermeira. Doutora em Enfermagem. Professora da Escola Paulista de Enfermagem da Universidade Federal de São Paulo. São Paulo (SP), Brasil. Email: suelyzanei@unifesp.br

ii Enfermeira. Graduada pela Escola Paulista de Enfermagem da Universidade Federal de São Paulo. São Paulo (SP), Brasil. Email: rakeloliveira@hotmail.com

iii Enfermeira. Doutora em Enfermagem. Professora da Escola Paulista de Enfermagem da Universidade Federal de São Paulo. São Paulo (SP), Brasil. Email: iveth.whitaker@unifesp.br
} 
Resumen: Objetivo: caracterizar los profesionales de programas de Residencia Multiprofesional a partir de dados sociodemográficos, educacionales y rutinas de vida como sueño y hábitos alimentares y evaluar su calidad de vida (CV). Método: estudio descriptivo, transversal, cuantitativo realizado en una universidad pública en el inicio del 2014. El instrumento utilizado para evaluación de calidad de vida fue el Word Health Organization Quality of Life-bref (WHOQOL-bref), aplicado por la plataforma electrónica. Resultados: la confiabilidad del instrumento varió de 0,63-0,79. Participaron 49\% del total de residentes matriculados, la mayoría mujeres, con 25 años, solteras, procedentes de São Paulo. Los profesionales residentes duermen en media seis horas por noche y refieren alimentación más o menos saludable. La puntuación de CV en el dominio Físico fue 55,96; Psicológico 56,96; Relaciones Sociales 62,76 y Medio Ambiente 49,65. Conclusión: la evaluación del WHOQOL-bref reveló que, en la mayoría de los dominios, la CV de los residentes es comprometida.

Descriptores: Calidad de vida; Estudiantes del área de la salud ; Empleos en salud ; Capacitación profesional

\section{Introdução}

O Programa Nacional de Bolsas para Residências Multiprofissionais e em Área Profissional da Saúde foi instituído a partir da publicação da Portaria Interministerial nº 1.077, de 12 de Novembro de $2009^{1}$ e, a partir de 2010, diversos programas foram iniciados pelo país, a maioria, vinculados às universidades federais e homologados pelo Ministério da Educação e Cultura. Em curto espaço de tempo houve aumento expressivo do número de bolsas, sendo que no período de 2010 - 2014 houve incremento de 700\%. O contingente de residentes multidisciplinares nesse período passou de pouco mais de 400 para mais de 3.200 residentes. $^{2}$

Os programas multidisciplinares foram moldados à semelhança dos programas de residência médica com valor da bolsa e carga horária idênticos, visando o treinamento profissional, predominantemente, em serviço. O residente cumpre 60 horas semanais, das quais $80 \%$ correspondem às atividades práticas e $20 \%$ às atividades teóricas. ${ }^{1}$

Apesar do modelo consagrado da residência médica no que diz respeito à ênfase nas atividades práticas executadas em 60 horas semanais, há muito se questiona o impacto da extensa carga horária sobre a saúde física, mental e qualidade de vida do residente médico. ${ }^{3}$ Enquanto estudantes, a qualidade de vida $(\mathrm{QV})$ comprometida pode afetar o aprendizado, a 
concentração ou a motivação para os estudos. Os profissionais/estudantes da saúde não são exceções, conforme apontam alguns estudos. ${ }^{3-4}$

Destaca-se que apesar do profissional residente (médico ou multiprofissional) não manter vínculo empregatício formal, suas atividades laborais são semelhantes ou até mais exaustivas do que os demais trabalhadores da mesma área profissional. Entretanto, o residente é considerado estudante de pós-graduação - visando atingir as competências exigidas pela profissão - e não trabalhador, a despeito de desempenharem atividades laborais em $80 \%$ de sua carga horária obrigatória, que os predispõem tanto a riscos ocupacionais quanto em responsabilidade civil profissional. ${ }^{5}$

Considerando-se que os residentes estão em processo de aprimoramento profissional, com ênfase nas atividades práticas; que diariamente estão em contato direto com os pacientes; que as condições de vida podem influenciar no processo de aprendizagem e na assistência prestada, a questão que norteou o presente estudo foi qual a qualidade de vida dos profissionais matriculados nos programas?

Assim sendo, os objetivos foram caracterizar os profissionais dos programas de Residência Multiprofissional quanto aos dados sociodemográficos, educacionais e rotinas de vida diária como sono e hábitos alimentares e avaliar sua qualidade de vida (QV).

\section{Método}

Trata-se de estudo descritivo, transversal, com abordagem quantitativa realizado em um hospital universitário, vinculado a uma universidade federal, localizado na cidade de São Paulo.

Os dados foram coletados em janeiro e fevereiro de 2014 e a amostra foi composta por 128 dos 263 residentes, de diversos programas e profissões, que concordaram em participar do estudo. 
Qualidade de vida dos profissionais de saúde dos programas de residências multidisciplinares | 4

Os questionários foram transformados em formulários eletrônicos e enviados para o email de cada residente para que pudessem acessá-los e responder online. A escolha por esta estratégia de aplicação foi possibilitar ao respondente o acesso a qualquer momento e quando julgasse conveniente. Considerou-se também, a facilidade do uso da internet pela maioria e a possibilidade de abordagem de todos os residentes em curto período de tempo. Entretanto, como não é comum a aplicação de questionários relacionados à QV por via eletrônica no nosso meio, optou-se por verificar a confiabilidade do instrumento aplicado à amostra.

O recurso, plataforma Google Docs, está disponível gratuitamente e pode ser utilizado sem restrições. O aplicativo possibilita criar formulários que são acessados pelos participantes e, após o seu preenchimento, o respondente os envia e estes ficam disponíveis online para que os responsáveis pela criação dos formulários possam acessá-los. O aplicativo permite também que os resultados possam ser ou não visualizados pelos demais participantes. No presente estudo, todas as informações do banco eletrônico ficaram disponíveis apenas para os pesquisadores responsáveis.

Cada participante preencheu dois questionários enviados separadamente, sendo que o primeiro continha questões sobre o perfil sociodemográfico, estudantil e rotinas da vida diária (quantidade de horas de sono diária e qualidade da alimentação). O segundo foi o instrumento específico de avaliação de QV, World Health Organization Quality Of Life-bref (WHOQOLbref). Ambos continham um lembrete para responder o outro questionário e as respectivas orientações para o preenchimento. A primeira pergunta tratava da concordância em participar do estudo, que sendo afirmativa, permitia ao respondente acesso às demais questões.

O questionário WHOQOL-bref é um instrumento de avaliação da QV geral desenvolvido pela Organização Mundial de Saúde (OMS), que constitui a versão abreviada do WHOQOL-100. Os instrumentos foram elaborados a partir da premissa adotada pela OMS de que "qualidade de vida é a percepção do indivíduo de sua posição na vida no contexto da cultura 
e sistema de valores nos quais ele vive e em relação aos seus objetivos, expectativas, padrões e preocupações”. Para os estudiosos do tema, a QV não pode ser avaliada levando-se em consideração apenas um aspecto de vida, como saúde ou condições de trabalho. Assim, a QV deve ser mensurada considerando-se que é um construto multidimensional e que a vida dos indivíduos é multifacetada, composta por diversos domínios. ${ }^{6}$

O WHOQOL-bref é composto por 26 questões, das quais as duas primeiras são genéricas e questionam sobre como o indivíduo avalia sua QV (Q1) e a segunda (Q2), o quão ele está satisfeito com sua saúde. As demais são distribuídas em quatro domínios: Físico, Psicológico, Relações Sociais e Meio Ambiente, totalizando 24 itens. Para cada questão, o participante escolhe uma alternativa relacionada ao grau de intensidade, capacidade, frequência ou avaliação, de acordo com o tipo da pergunta. Todas são graduadas em uma escala de Likert que varia de 1 a 5 pontos. Cada opção tem sua valoração correspondente, a maioria crescente, e questões específicas possuem valores invertidos. Os escores finais da QV são pontuados por domínio de forma independente, não havendo escore único. A determinação dos escores dos domínios é realizada multiplicando-se a média de todos os itens incluídos dentro de um domínio por quatro. Assim sendo, o escore pode variar de zero a 100, sendo que quanto maior o valor, melhor é o domínio de qualidade de vida avaliado. ${ }^{6}$

Em relação à análise das demais variáveis, para as contínuas calculou-se média, desvio padrão, mediana, mínimo e máximo. Para as variáveis categóricas calculou-se frequência e percentual. Para correlacionar as variáveis com seus respectivos domínios e cada domínio com as questões Q1 e Q2 utilizou-se o coeficiente de correlação de Spearman. Em relação à análise da confiabilidade do instrumento, verificou-se a consistência interna por domínio através do Alpha de Cronbach, considerando-se $\alpha \leq 0,30$ confiabilidade muito baixa, $\alpha$ entre 0,31 - 0,59 baixa, 0,60 - 0,75 moderada, 0,76-0,90 alta. $^{7}$ Adotou-se um nível de significância de 5\% (p-valor $\leq$ $0,05)$. 
Qualidade de vida dos profissionais de saúde dos programas de residências multidisciplinares I 6

O estudo foi aprovado em 08/01/2014 pelo Comitê de Ética em Pesquisa em Seres Humanos da instituição (Parecer 507.856, CAAE 25317013.4.0000.5505) respeitando os aspectos éticos da Resolução nº 466/2012 do Conselho Nacional de Saúde.

\section{Resultados}

Dos 263 residentes multiprofissionais inscritos nos programas de residência da referida universidade, 109 responderam as perguntas referentes às características sociodemográficas e outros dados de interesse, representando $42 \%$ do total.

Em relação aos dados sociodemográficos e educacionais, verificou-se predomínio de residentes do sexo feminino, com média de 25 anos, solteiros, procedentes de faculdades públicas e moradores na capital. Dados mais específicos encontram-se na Tabela 1.

Tabela 1- Distribuição dos residentes multiprofissionais segundo dados sociodemográficos, socioprofissionais e educacionais. São Paulo, 2014

\begin{tabular}{lcc}
\hline Variáveis $(\mathrm{n}=109)$ & $\mathbf{n}$ & $\mathbf{\%}$ \\
\hline Sexo & & \\
Feminino & 100 & 91,75 \\
Faixa etária (anos) & & \\
20 a 25 & 69 & 63,31 \\
26 a 30 & 36 & 33,03 \\
31 a 35 & 2 & 1,83 \\
36 a 40 & - & - \\
$>40$ & 2 & 1,83 \\
Estado Civil & & \\
Casado & 9 & 8,26 \\
Solteiro & 98 & 89,9 \\
Outro & 2 & 1,84 \\
Área profissional & & \\
Assistência Social & 8 & 7,34 \\
Enfermagem & 27 & 24,77 \\
Farmácia & 9 & 8,26 \\
Fisioterapia & 17 & 15,6 \\
Fonoaudiologia & 9 & 8,26 \\
Nutrição & 11 & 10,09 \\
\hline
\end{tabular}


7 | Zanei SSV, Oliveira RA, Whitaker IY

\begin{tabular}{lcc}
\hline Psicologia & 22 & 20,18 \\
Não Responderam & 6 & 5,5 \\
Instituição na qual se formou (graduação) & & \\
Privada & 48 & 44,03 \\
Pública & 61 & 55,97 \\
Tempo de formado (anos) & & \\
1 a 1,5 & 45 & 41,29 \\
2 a 2,5 & 43 & 39,45 \\
3 a 3,5 & 14 & 12,84 \\
$\geq 4$ & 7 & 6,42 \\
Procedência & & \\
São Paulo - Capital & 54 & 49,54 \\
Outro município/Estado & 55 & 50,46 \\
Com quem mora & & \\
Esposo (a) ou Companheiro (a) & 13 & 11,93 \\
Pais & 43 & 39,44 \\
Colegas & 32 & 29,36 \\
Sozinho & 13 & 11,93 \\
Outro & 8 & 7,34 \\
Ajuda financeira além da bolsa & & \\
Não & 87 & 79,92 \\
\hline
\end{tabular}

Quanto ao número de horas de sono, do total de respondentes (109), 31 residentes $(28,44 \%)$ informaram dormir até 5 horas por noite, 56 (51,38\%) dormem em torno de 6 horas e 22 (20,18\%) referiram dormir de 7 a 8 horas diariamente. Em relação à alimentação e de acordo com a autopercepção, 15 (13,76\%) assinalaram que se alimentam de forma saudável, 52 (47,70\%) assinalaram a opção mais ou menos e $42(38,54 \%)$ optaram pela alternativa negativa.

Em relação ao questionário de QV, dos 263 residentes multiprofissionais inscritos nos programas de residência, 128 responderam as questões do WHOQOL-bref, representando 49\% do total.

Em relação à Q1 “Como você avalia sua QV?”, e a Q2 “quão satisfeito você está com sua saúde?” os percentuais apresentados estão descritos na tabela 2. 
Qualidade de vida dos profissionais de saúde dos programas de residências multidisciplinares | 8

Tabela 2 - Análise descritiva das questões 1 e 2 do WHOQOL bref. São Paulo, 2014

\begin{tabular}{llll}
\hline $\begin{array}{l}\text { Q1. Como você avalia sua QV? } \\
(\mathrm{n}=128)\end{array}$ & $\begin{array}{l}\text { Q2. Quão satisfeito você está com sua saúde? } \\
(\mathrm{n}=263)\end{array}$ & $\mathrm{n}(\%)$ \\
\hline Opção & $\mathrm{n}(\%)$ & Opção & $10(7,8)$ \\
\hline Muito ruim & $5(3,9)$ & Muito insatisfeito & $44(34,4)$ \\
Ruim & $35(27,3)$ & Insatisfeito & $25(19,5)$ \\
Nem ruim, nem boa & $46(35,9)$ & Nem satisfeito, nem insatisfeito & $45(35,2)$ \\
Boa & $37(28,9)$ & Satisfeito & $4(3,1)$ \\
Muito Boa & $5(3,9)$ & Muito Satisfeito & \\
\hline
\end{tabular}

Destaca-se, ainda, que a confiabilidade do instrumento aplicada aos participantes por via eletrônica, considerando-se os quatro domínios, através do Alfa de Cronbach foi de 0,70. Em relação aos escores de QV, as médias dos domínios estão descritas na tabela 3. As questões Q1 e Q2 apresentaram correlações significativas em todos os domínios, com valor de $r$ de 0,42 a 0,65 $(\mathrm{p}<0,0001)$.

Tabela 3 - Escores médios dos domínios de qualidade de vida-WHOQOL-bref segundo residentes multiprofissionais. São Paulo, 2014

\begin{tabular}{lcccc}
\hline \multicolumn{1}{c}{ Domínio QV } & Físico & Psicológico & $\begin{array}{c}\text { Relações } \\
\text { Sociais }\end{array}$ & $\begin{array}{c}\text { Meio } \\
\text { Ambiente }\end{array}$ \\
\hline Escore médio & 55,96 & 56,95 & 62,76 & 49,65 \\
Alfa de Cronbach & 0,77 & 0,79 & 0,63 & 0,64 \\
\hline
\end{tabular}

\section{Discussão}

A amostra foi constituída predominantemente por profissionais do sexo feminino, jovens e solteiros. Considerando-se que as mulheres representam o maior número de estudantes universitários no país, o resultado reflete tal realidade. ${ }^{8}$

As profissões representadas nos programas foram enfermagem, farmácia, fisioterapia, fonoaudiologia, nutrição e psicologia, das quais enfermagem e fisioterapia apresentaram o maior número de participantes. Tal fato justifica-se por serem as profissões contempladas com o maior número de vagas na maioria dos programas.

Vale destacar que em 2014 a instituição ofereceu 151 vagas para os programas multiprofissionais distribuídas entre as várias profissões de saúde (edital 710/2013). ${ }^{9}$ O total de 
candidatos que efetivamente prestaram o processo seletivo foi de 753 , resultando em média cinco candidatos por vaga (dados calculados a partir de informações aos coordenadores de programa de residência da instituição). Tal proporção evidencia a procura pelos programas de residência multiprofissionais por jovens recém-graduados que podem sentir-se atraídos pela possibilidade de realizarem uma especialização na sua área profissional. O atrativo adicional pode ser o recebimento da bolsa financiada no valor de $\mathrm{R} \$ 3330,00$ (2018) que, em algumas profissões é superior ao salário inicial oferecido pelo mercado de trabalho. De acordo com uma instituição de empregos, o salário médio de um nutricionista no Brasil é de $\mathrm{R} \$ 2.447,20$; o fisioterapeuta recebe em média $R \$ 2188,00$ e o enfermeiro $R \$ 3092,00$, para fins de exemplificação. ${ }^{10}$ A carga horária de 60 horas semanais, ${ }^{1}$ a princípio, parece não interferir na busca pelos programas de residência.

Cerca da metade dos residentes era procedente da capital e a maioria residia na casa dos pais. Entretanto, a outra metade era do interior ou de outro Estado, demonstrando que São Paulo continua a atrair profissionais de outras regiões para trabalho e estudos. Tal fato, provavelmente, é devido ao Estado, particularmente a capital, ser considerado um dos mais desenvolvidos do país, com os melhores indicadores de Índice de Desenvolvimento Humano e destaque na área de educação. ${ }^{11}$

Em relação aos hábitos diários, um resultado preocupante e que pode comprometer a saúde e o desempenho geral dos residentes é que a maioria (aproximadamente 80\%) dorme seis ou menos horas por noite e aproximadamente $65 \%$ deles assinalaram as alternativas “insatisfeitos" ou "muito insatisfeitos" com o sono. Um estudo que avaliou a normalidade do sono comprova que a necessidade diária entre adultos jovens saudáveis está entre 7-9 horas de sono. ${ }^{12}$ Além disso, as poucas horas de sono por noite pode ser um fator importante para a piora das condições de saúde e bem estar e, sua privação é fator contribuinte para sonolência diurna, desânimo, sintomas depressivos, entre outros. ${ }^{13}$ A sonolência excessiva foi também um dos 
Qualidade de vida dos profissionais de saúde dos programas de residências multidisciplinares I 10

problemas identificados em uma revisão bibliográfica entre os residentes médicos, além de bournout, estresse, fadiga, dificuldades de enfrentamento relacionadas as condições de vida e de trabalho e relação entre carga horária de trabalho e qualidade de vida. ${ }^{3}$

Outro estudo relevante sobre a privação do sono entre médicos residentes em UTI e unidade coronariana mostrou que aqueles que mantiveram o esquema tradicional de horas de plantões (24 horas ou mais) tenderam a apresentar maior índice de erros durante a prática diária do que aqueles que realizaram plantões mais curtos. Tal resultado mostra a importância do sono e descanso para minimizar os possíveis erros durante o plantão. ${ }^{14}$ Apesar de não ser permitido plantões que excedam 12 horas sem descanso na residência multidisciplinar da instituição estudada, a carga horária semanal total é a mesma do residente médico, ambas superiores à carga horária legal de trabalhadores em geral. Da mesma forma que qualquer trabalhador que tenha carga horária extensa associada principalmente a privação do sono, a possibilidade de erros na execução de tarefas e/ou acidentes no trabalho são eventos amplamente conhecidos entre os profissionais de saúde. ${ }^{15}$

Em relação aos hábitos alimentares, de acordo com a autopercepção, apenas 15 residentes (13,76\%) assinalaram que se alimentam de forma saudável. Quase metade dos respondentes, ou seja, $52(47,70 \%)$ assinalou a opção “mais ou menos” e $42(38,54 \%)$ responderam que não se alimentam adequadamente. Vale esclarecer que todos os residentes têm direito a alimentação gratuita (café da manhã e almoço) na própria instituição e a maioria almoça regularmente no refeitório destinado aos residentes médicos e multidisciplinares.

Tal como os participantes do presente estudo, jovens e estudantes, em geral, não consideram suas práticas alimentares adequadas, inclusive entre alunos da área de saúde. ${ }^{16} \mathrm{~A}$ alimentação pouco adequada entre a população de adultos jovens é um fenômeno disseminado, constatado também entre universitários italianos ${ }^{17}$ e libaneses. ${ }^{18}$ A inadequação quanto aos hábitos alimentares entre discentes e recém graduados, pode ser devido a diversos fatores entre 
os quais problemas financeiros, horários irregulares ou desconhecimento sobre recomendações nutricionais. ${ }^{19}$

Vale salientar que práticas alimentares adequadas se somam a outros fatores que podem minimizar ou prevenir o surgimento de diversas doenças, no tempo presente (infecções agudas) e futuro (doenças crônicas). ${ }^{20}$ Entre os próprios acadêmicos é clara a relação entre bom estado de saúde (incluindo-se boa alimentação) e bom desempenho acadêmico. ${ }^{21}$

No que diz respeito aos resultados da aplicação do questionário de QV, o WHOQOLbref apresentou boa confiabilidade por meio do formulário eletrônico, com Alpha de Cronbach maior do que 0,70 nos domínios Físico e Psicológico e nos domínios Relações Sociais e Meio Ambiente os valores foram de 0,63 e 0,64, respectivamente. Para os dois primeiros, os resultados são considerados satisfatórios e nos dois últimos apesar de alcançarem valores mais baixos, são considerados aceitáveis. Tais valores se justificam, pois, o domínio relações sociais possui menor número de itens e o domínio meio ambiente engloba diferentes elementos no mesmo domínio, tais como ambiente físico, segurança, transporte, recursos financeiros e disponibilidade de novas informações. As características dos domínios (poucos itens ou vários elementos no mesmo domínio) tendem a diminuir o valor do Alfa de Cronbach. ${ }^{22}$ Os resultados do teste de confiabilidade confirmaram que o instrumento foi válido e confiável para avaliação da QV da amostra do estudo.

Em relação aos escores de QV propriamente dito, no domínio físico o escore médio foi de 55,96 pontos. Considerando-se que a escala pode variar de zero a 100 e que quanto maior o valor, melhor é qualidade de vida no domínio, o resultado revela que a QV-domínio físico é relativamente baixa e provavelmente, tal fato se deve aos itens "satisfação com o sono" e a “quantidade de energia diária”. Essas tiveram respostas com conotações negativas, a saber, 64,8\% estão 'insatisfeitos” com o sono e 90,6\% responderam que possuem "média quantidade, 
Qualidade de vida dos profissionais de saúde dos programas de residências multidisciplinares | 12

nada ou muito pouca energia”. O número de respondentes que escolheram tais alternativas pode ter contribuído para a redução da média global do domínio.

O escore do domínio físico da amostra é próximo ao de um estudo realizado entre aprimorandos e aperfeiçoandos de cursos de saúde não médicos utilizando o WHOQOL-100 em uma cidade do interior paulista, que também apresentou valores próximos a 60,0. ${ }^{23}$ Apesar do estudo citado não mencionar a carga horária dos cursos, parece que as atividades estudantis na área da saúde que envolvem estágios práticos contribuem para o cansaço ou fadiga e distúrbios de sono.

A ocorrência de transtornos mentais comuns (insônia, fadiga, irritabilidade, esquecimento, dificuldade de concentração e queixas somáticas) é relevante entre os residentes da área da saúde. Estudo realizado entre residentes médicos e não médicos, revelou que entre os médicos os sintomas são mais intensos, pressupondo-se comprometimento na qualidade de vida desses jovens profissionais. ${ }^{24}$

Quanto ao domínio Psicológico avaliado pelo WHOQOL-bref, o escore médio também foi relativamente baixo, de 56,96 pontos. Os itens do domínio estão relacionados à presença de sentimentos positivos ou negativos em relação a si próprios e a vida, capacidade de concentração e autoimagem. Chama a atenção as alternativas assinaladas, por exemplo, para a questão "com que frequência você tem sentimentos negativos tais como mau humor, desespero, ansiedade, depressão”, 57,8\% dos respondentes assinalaram as opções “frequentemente”, “muito frequentemente” e “sempre”. A opção “às vezes” foi selecionada por $39,8 \%$.

Cerca de $2 / 3$ dos residentes consideram que aproveitam "nada”, "muito pouco" ou "mais ou menos” a vida e, igualmente, a capacidade de concentração encontra-se em patamares similares. Tais respostas provenientes de uma população de jovens denota que o cotidiano tem sido árduo, comprometendo, provavelmente, também o aprendizado, considerando-se a dificuldade de concentração da maioria. Ainda, $40 \%$ estão "satisfeitos" consigo mesmo e $52 \%$ 
acham que "a sua vida tem sentido". Um estudo realizado com residentes médicos ${ }^{24}$ e outro com os de enfermagem, ${ }^{25}$ ambos com o SF-36 (Medical Outcomes Study 36 - Item Short - Form Health Survey), mostrou que os escores em Saúde Mental, que se assemelha ao domínio Psicológico do WHOQOL-bref, variaram entre 44 e 56 pontos (escore de zero-100). Estes valores aproximam-se aos obtidos no presente estudo $(56,96)$, mas são adversos quando comparados ao do estudo realizado com população geral de indivíduos saudáveis na região Sul do Brasil, que evidenciou média de 73,82 pontos no domínio. ${ }^{26}$ Cita-se ainda, em consonância com o presente estudo, pesquisa que avaliou estresse entre residentes multidisciplinares em instituição pública, na qual foi constatado que $48,65 \%$ dos respondentes apresentavam alto índice de estresse durante a residência, demonstrando que o aspecto emocional tem impacto importante na vida dos profissionais. ${ }^{27}$

O domínio Relações Sociais foi o que apresentou maior escore médio, com 62,76 pontos, apesar dos residentes terem se queixado de falta de tempo para realização de outras atividades não relacionadas à vida acadêmica. Porém, o valor, apesar de melhor, ainda está longe de ser considerado de boa qualidade no aspecto social. Os itens que compõem esse domínio abordam temas sobre relações pessoais, vida sexual e apoio de amigos. Apesar da opção "satisfeito" ter sido escolhida pela maioria dos participantes para classificar tais aspectos de suas vidas, nas demais perguntas ("satisfação com suas relações pessoais - amigos, parentes, conhecidos, colegas)" e "satisfação com sua vida sexual”), os percentuais não alcançaram $50 \%$. A segunda opção mais assinalada foi "muito satisfeito" em relação ao apoio de amigos, revelando que a amizade desenvolvida entre os residentes, é a questão a mais positiva do domínio Relações Sociais.

Comparando-se o escore obtido nesse estudo $(62,76)$ com o do estudo realizado com a população geral $(78,30),{ }^{26}$ as relações sociais mostraram-se inferiores no grupo de residentes multiprofissionais. Entretanto, se comparado com as médias dos escores dos residentes médicos 
Qualidade de vida dos profissionais de saúde dos programas de residências multidisciplinares I 14

$(56,25)$ e dos de enfermagem $(50,0)$, as médias dos residentes multiprofissionais são relativamente superiores. ${ }^{24-25} \mathrm{O}$ valor mais alto pode ser justificado pela proximidade entre os profissionais favorecido pela multidisciplinaridade. Além disso, o fato de permanecerem em constante contato durante várias horas do dia pode favorecer a formação de vínculos afetivos e sociais entre eles.

O domínio Meio Ambiente foi o que apresentou o escore mais baixo com média de 49,65 pontos. Levando-se em conta que estudo foi realizado em São Paulo, uma metrópole agitada, com elevados níveis de poluição e barulho, alto custo de vida e violência; não causa surpresa os resultados obtidos no domínio. Os temas abordados são segurança, ambiente físico e condição financeira, que tenderam a respostas negativas, justificando seu baixo valor. A pior resposta foi referente ao clima, poluição, barulho e atrativos, com $39,8 \%$ dos residentes considerando o ambiente físico no qual se encontravam "muito pouco" ou "mais ou menos" saudável. O item sensação de segurança obteve $50 \%$ de respostas apontando para "mais ou menos" e satisfação monetária obteve 53,5\% de respostas indicando "média satisfação", com $80 \%$ dos participantes referindo não receber ajuda além da bolsa do programa. Quanto à locomoção, apesar da maioria dos participantes se locomoverem a pé $(44,95 \%)$ ou por transporte público $(80,74 \%), 39,1 \%$ indicou estar "satisfeito" com o meio de transporte utilizado.

Esse resultado (escore total do domínio de 49,65 ) é mais baixo do que o obtido no estudo com aprimorandos e aperfeiçoandos (média de 59,04), realizado no interior de São Paulo. ${ }^{21}{ }^{23} \mathrm{~A}$ análise desse dado sugere que, em cidades afastadas da capital, a QV relacionada ao meio ambiente parece ser um pouco mais favorável.

Os escores relativamente baixos para todos os domínios refletem a avaliação geral sobre a QV (questão 01 do WHOQOL-bref), na qual somente 32,8\% a consideravam "boa” ou "muito boa” e a maioria $(35,9 \%)$ optou por “nem ruim, nem boa”. A mesma situação aconteceu com a 
questão 2 "quão satisfeito você está com sua saúde?", que apresentou um percentual de “satisfeitos” e “insatisfeitos” próximos de 38,3\% e 42,2\%, respectivamente.

Apesar do número de respondentes não refletir a totalidade dos residentes e em razão de representarem apenas uma instituição entre as muitas que oferecem programas de residência multidisciplinar os participantes pertenciam a oito dos 12 programas da instituição e representaram mais de $50 \%$ dos residentes dos seus respectivos programas. Assim, os baixos valores dos escores de QV devem ser considerados preocupantes pelos coordenadores, levandose em conta que além de prejudiciais para a vida e saúde dos residentes, tal condição pode comprometer seriamente o desempenho acadêmico e a formação profissional, além de oferecer riscos à segurança dos usuários/clientes durante a assistência.

Frente a tais resultados sugere-se que gestores de instituições de saúde nas quais há programas de residência preocupem-se com a saúde de seus residentes médicos e multiprofissionais, pois estes estão em contato direto com os usuários e podem comprometer a qualidade do atendimento.

Quanto aos coordenadores e responsáveis pelos programas, é premente que reflitam profundamente sobre a viabilidade de novas estratégias que possam ser implementadas no dia a dia dos residentes, tais como moradia, facilidades para as refeições e locais para períodos de descanso apropriados sem, contudo, descaracterizar a essência dos programas. Além disso, os próprios residentes e futuros ingressantes podem ser orientados desde o inicio dos cursos sobre os recursos oferecidos pelas instituições que possam auxiliá-los no controle de saúde física e mental, tais como atendimento nos ambulatórios/clínicas de saúde que incluam os residentes.

Além disso, estudos relacionados à vida acadêmica, profissional e de QV poderão subsidiar as instituições de saúde e coordenadores de programa a avaliarem e planejarem atividades que minimizem possíveis repercussões negativas no cotidiano institucional, incluindo-se o desempenho profissional do residente. 
Qualidade de vida dos profissionais de saúde dos programas de residências multidisciplinares I 16

\section{CONCLUSÃO}

Os residentes multidisciplinares dormem menos do que o recomendado para a faixa etária e a maioria acredita que não se alimenta adequadamente.

Em relação à qualidade de vida dos residentes multidisciplinares, os escores de QV mensurados por meio do WHOQOL-bref foram relativamente baixos em todos os domínios, sendo o pior o de Meio Ambiente e o melhor o de Relações Sociais.

Apesar da pesquisa englobar residentes de programas distintos nas diversas áreas de atendimento hospitalar, como limitações do presente estudo cita-se a realização em uma única universidade, retratando, portanto, uma realidade particular. Entretanto, há que se considerar que o número de programas e o quantitativo de residentes são expressivos e que no contexto geral muito se assemelha a diversos programas do país que seguem as normativas gerais definidas pelo Ministério da Educação (MEC).

Assim, considerando-se as implicações para o dia a dia dos residentes e para a prática profissional, tais resultados são preocupantes, pois podem resultar em prejuízos na qualidade dos processos de aprendizagem e aquisição de competências. Ao mesmo tempo, e igualmente relevante, por tratar-se de curso eminentemente prático, pode comprometer a segurança e a qualidade de assistência prestada pelos residentes aos clientes/pacientes. Para a instituição e sistemas de saúde, o desempenho comprometido dos residentes pode prejudicar a imagem institucional.

Finalizando, destaca-se ainda, a importância do presente estudo considerando-se a magnitude do crescimento dos programas de residência multiprofissional pelo país. Se por um lado, tal como ocorre com os residentes médicos, o treinamento em serviço aprimora as competências técnicas dos profissionais, o intenso desgaste à saúde física e mental pode gerar danos de difícil controle tanto momentâneos como persistentes que devem ser avaliados. Não é 
aceitável que no contexto da saúde, os profissionais que cuidam, não recebam a devida atenção as suas condições de saúde que afetam diretamente sua qualidade de vida.

\section{Referências}

1. Brasil. Ministério da Educação. Ministério da Saúde. Portaria Interministerial MEC/MS n. 1077, de 12 de novembro de 2009. Dispõe sobre a Residência Multiprofissional em Saúde e a Residência em Área Profissional da Saúde, e institui o Programa Nacional de Bolsas para Residências Multiprofissionais e em Área Profissional da Saúde e a Comissão Nacional de Residência Multiprofissional em Saúde [Internet]. 2012 [acesso em 2014 out 31]. Diário Oficial da União, Brasília (DF); 13 nov 2009. Seção 1, p. 7. Disponível em: http://portal.mec.gov.br/index.php?option=com_docman\&view=download\&alias=15462-por-1077$\underline{12 \text { nov-2009\&Itemid=30192 }}$

2. Brasil. Ministério da Educação. A democratização e expansão da educação superior no país 2003 - 2014 [Internet]. Brasília (DF); 2014 [acesso em 2015 jun 31]. Disponível em:

http://portal.mec.gov.br/index.php?option=com_docman\&view=download\&alias=16762-balanco-socialsesu-2003-2014\&Itemid=30192

3. Mordant P, Deneuve S, Rivera C, Carrabin N, Mieog JS, Malyshev N, et al. Quality of life of surgical oncology residents and fellows across Europe . J Surg Educ. 2014;71(2):222-8.

4. Garcia-Rodriguez LR, Sanchez DL, Ko AB, Williams AM, Peterson E, Yaremchuk KL. A study of otolaryngology resident quality of life and sleepiness. Laryngoscope Investig Otolaryngol. 2017;2(3):113-8.

5. Lima, AMC. Residência médica sob a óptica do direito do trabalho. Rev ESMESC [Internet] 2010. [acesso em 18 out 2014];17(23):175-96. Disponível em: https://revista.esmesc.org.br/re/article/view/8/9.

6. Moreno AB, Faerstein E, Werneck GL, Werneck GL; Lopes CS; Chor D. Propriedades psicométricas do instrumento abreviado de avaliação de qualidade de vida da Organização Mundial da Saúde no estudo Pro-Saúde. Cad Saúde Pública. 2006; 22(12):2585-97.

7. Freitas ALP, Rodrigues SG. A avaliação da confiabilidade de questionários: uma análise utilizando o coeficiente alfa de Cronbach [Internet]. In: 12 Simpósio de Engenharia da Produção; 2005 nov 07-09; Bauru (SP). Bauru (SP): SIMPEP; 2005 [acesso em 2016 jun 21]. Disponível em:

https://www.researchgate.net/publication/236036099_A_avaliacao_da_confiabilidade_de_questionarios_u ma_analise_utilizando_o_coeficiente_alfa_de_Cronbach

8. Brasil. Ministério da Educação. Censo da Educação Superior 2014: Notas Estatísticas [Internet]. 2014 [acesso em 2016 jun 21]. Disponível em:

http://download.inep.gov.br/educacao_superior/censo_superior/documentos/2015/notas_sobre_o_censo_d a_educacao_superior_2014.pdf 
Qualidade de vida dos profissionais de saúde dos programas de residências multidisciplinares | 18

9. Universidade Federal de São Paulo. Pró-Reitoria de Extensão. Edital n. 710/2013, Processo Seletivo para R1 - 2014 [Internet]. São Paulo; 2013 [acesso em 2018 jan 15]. Disponível em:

https://www.unifesp.br/reitoria/proex/images/PROEX/coremu/processoseletivo2014/editais/rmu_edital_71 0_2013_processoseletivo_2014.pdf

10. Candidatos. Guia de Profissões e Salários. [Internet]. Brasil. [acesso em 2018 jan 15]. Disponível em: https://www.catho.com.br/profissoes/.

11. Instituto de Pesquisa Econômica Aplicada (IPEA). Programa das Nações Unidas para o Desenvolvimento (IPEA). O Índice de desenvolvimento humano municipal brasileiro. Série atlas do desenvolvimento humano no Brasil. Brasília; 2013 [acesso em 2016 jun 18]. Disponível em: http://www.ipea.gov.br/portal/images/stories/PDFs/130729_AtlasPNUD_2013.pdf

12. Hirshkowitz M, Whiton K, Albert SM, Alessi C, Bruni O, Don Carlos L, et al. National sleep foundation's sleep time duration recommendations: methodology and results summary. Sleep Health. 2015;1(1):40-3.

13. Santa ND, Cantilino A. Suicídio entre médicos e estudantes de medicina: revisão de literatura. Rev Bras Educ Méd [Internet]. 2016 dez [acesso em 2017 out 23];40(4):772-80. Disponível em: http://www.scielo.br/pdf/rbem/v40n4/1981-5271-rbem-40-4-0772.pdf

14. Anderson C, Sullivan JP, Flynn-Evans EE, Cade BE, Czeisler CA, Lockley SW. Deterioration of neurobehavioral performance in resident physicians during repeated exposure to extended duration work shifts. Sleep. 2012;35(8):1137-46.

15. Tsafrir Z, Korianski J, Almog B, Many A, Wiesel O, Levin I, et al. (2015). Effects of fatigue on residents' performance in laparoscopy. J Am Coll Surg. 2015;(221):564-70.

16. Loureiro MP. Estado nutricional e hábitos alimentares de universitários. Segur Aliment Nutri [Internet]. 2016 [acesso em 2017 out 24];23(2):955-72. Disponível em:

https://periodicos.sbu.unicamp.br/ojs/index.php/san/article/view/8647612/14849

17. Teleman AA, De Waure C, Soffiani V, Poscia A, Di Pietro ML. Nutritional habits in italian university students. Ann Ist Super Sanita. 2015;51(2):99-105.

18. Salameh P, Jomaa L, Issa C, Farhat G, Salamé J, Zeidan N, et al. Assessment of dietary intake patterns and their correlates among university students in Lebanon. Front Public Health [Internet]. 2014 out [acesso em 2017 out 31];2:185. Disponível em: https:/www.ncbi.nlm.nih.gov/pmc/articles/PMC4204443/ doi: 10.3389/fpubh.2014.00185

19. Wilson CK, Matthews JI, Seabrook JA, Dworatzek PDN. Self-reported food skills of university students. Appetite. 2017;108:270-6. 
20. Affonso CV, Sonati JG. Hábitos alimentares e prevenção de doenças. In: Vilarta R, organizador. Alimentação saudável, atividade física e qualidade de vida [Internet]. Campinas: Ipes; 2007 [acesso em 2016 jun 03]. Disponível em: http://www.fef.unicamp.br/fef/qvaf/alimentacao-saudavel-atividade-fisica-equalidade-de-vida

21. Peltzer K, Pengpid S. Health behaviour and self-reported academic performance among university students: an international study. Mediterr J Soc Sci. 2014;5(27):998-1005.

22. B Sharma. A focus on reliability in developmental research through Cronbach's Alpha among medical, dental and paramedical professionals. Asian Pac J Health Sci. 2016;3(4):271-8.

23. Lourenção LG, Moscardini AC, Soler ZASG. Qualidade de vida de residentes não médicos. Rev Enferm UFPE Online [Internet]. 2013 nov [acesso em 2014 out 31].7(11):6336-45. Disponível em: http://www.revista.ufpe.br/revistaenfermagem/index.php/revista/article/viewFile/2958/pdf_3852

24. Carvalho CN, Melo-Filho DA, Carvalho JAG, Amorim ACG. Prevalência e fatores associados aos transtornos mentais comuns em residentes médicos e da área multiprofissional. J Bras Psiquiatr. 2013;62(1):38-45

25. Franco GP, Barros ALBL, Nogueira-Martins LA. Qualidade vida e sintomas depressivos em residentes de enfermagem. Rev Latinoam Enferm. 2005;13(2):139-44.

26. Cruz LN, Fleck MPA, Oliveira MR, Camey AS. Hoffmann JF, Bagattini AM, et al. Health-related quality of life in Brazil: normative data for the SF-36 in a general population sample in the south of the country. Ciênc Saúde Colet. 2013;18(7):1911-21.

27. Silva R, Goulart C, Lopes L, Serrano P, Guido L. Estresse e hardiness entre residentes multiprofissionais de uma universidade pública. Rev Enferm UFSM. 2014;4(1):87-96.

\section{Autor correspondente}

Suely Sueko Viski Zanei

E-mail: suelyzanei@unifesp.br

Endereço: R. Napoleão de Barros, 754 . V. Clementino. São Paulo, SP.

CEP: 04024-002

\section{Contribuições de Autoria}

Autor 1 - Suely Sueko Viski Zanei

Contribuições: idealizadora e responsável do projeto de pesquisa, orientação do projeto e revisora do texto final. ORCID: https://orcid.org/0000-0003-2458-6862

Autor 2 - Raquel Araujo Oliveira 
Qualidade de vida dos profissionais de saúde dos programas de residências multidisciplinares I 20

Contribuições: co-autora do projeto, responsável pela construção e aplicação dos instrumentos de coleta na plataforma Google e contribuição na elaboração do texto do artigo.

ORCID: https://orcid.org/0000-0003-0184-0231

Autor 3 - Iveth Yamaguchi Whitaker

Contribuições: co-orientadora do projeto, contribuição na elaboração do texto do artigo e revisora do texto final.

ORCID: https://orcid.org/0000-0003-4431-6579

\section{Como citar este artigo}

Zanei SSV, Oliveira RA, Whitaker IY. Qualidade de vida dos profissionais de saúde dos programas de residências multidisciplinares. Rev. Enferm. UFSM. 2019 [Acesso em: Anos Mês Dia];vol e2: P1-P22. DOI:https://doi.org/10.5902/2179769230013 